\title{
Rare Presentation of Hookworm Infection
}

\author{
Parveen Malhotra*, Vani Malhotra, Sanjay Marwaha, Abhishekh, Pushkar and Yogesh Sanwariya \\ Department of Medical Gastroenterology, PGIMS, India
}

Submission: January 01, 2021; Published: January 29, 2021

*Corresponding author: Parveen Malhotra, Department of Medical Gastroenterology, PGIMS, Rohtak, India

Abstract

Objective: Hookworm is a pan global, easily treatable worm infestation, commonly seen in underdeveloped countries, manifests with variable presentations and proper diagnosis \& treatment can effectively reduce morbidity associated with it.

Clinical Presentation: We present a young female of 18 years who was admitted to the hospital with symptoms of pain abdomen, fever, and passage of worms from umbilicus. The diagnosis of hookworm infection was confirmed on the basis of presence of ova on stool examination and microscopic examination of live worms which were coming out from umbilicus.

Conclusion: Hookworm passage from various natural openings of body like oral cavity, anal canal, nostrils, and umbilicus has been documented in literature but this is first case report in which multiple live hookworms' passage from umbilicus is being reported.

Keywords: Ancylostoma duodenale; Neactor americanus; Hookworm; Ova; Iron deficiency anemia

\section{Introduction}

Hookworm infection is a public health problem, caused by Ancylostoma duodenale and Neactor americanus. It is commonly seen in developing countries and characterized by iron deficiency anemia and hypoalbuminemia [1]. The patients usually present classically with pain abdomen, diarrhea, fever, loss of appetite and generalized weakness. In certain group of patients, migration of worm into different organs like brain, eye, lungs etc. can lead to symptoms depending upon the system involved. In selected cases, the worms can be seen exiting the human body from natural orifices, mostly the anal canal but rarely it can be seen to crawling out from nostrils, oral cavity, or umbilicus. The definitive diagnosis is made on the basis of identification of characteristic ova in the stool [2] and orally available anthelmintics given for short duration are effective in curing it but measures for preventing future re-infection are mandatory.

\section{Case Report}

An 18-year-old female, a known case of Koch's chest and was treated completely for it 12 years back. She had history of passage of two worms with per rectally ten years back. Now she presented with intermittent pain abdomen for last one year for which was being treated with oral and intravenous analgesics by local practitioners but was never fully investigated for the same. She had single episode of small amount of hematemesis two months back which was symptomatically treated as upper Gastrointestinal endoscopy done at that time was normal. After few days of this hematemesis episode, she developed passage of frothy fluid with feculent smell from umbilicus and she has to change her dress three-four times per day due to repeated wetting of her clothes. Subsequently her pain abdomen increased and was mainly Periumblical and associated with anorexia followed by passage of worms from umbilicus along with itching, fever for last six weeks. She had one more episode of upper gastrointestinal bleed at home and upper gastrointestinal endoscopy done after a gap of one week when she was admitted in our hospital was normal. She also gave history of irregular menstrual cycles, gynecological evaluation for which was normal, and it was attributed to anemia. On physical examination, the patient was conscious, co-operative, in discomfort, febrile and anemic. The systemic examination including chest, cardiovascular, central nervous system, ophthalmological and dermatological was normal. The complete haemogram revealed hemoglobin of 9.6 $\mathrm{g} / \mathrm{dL}$, white blood cell counts 13,200/L, microcytic hypochromic anemia with no malaria parasite. The renal \& liver function test, blood sugar, serum amylase \& electrolytes, serum vitamin B12, D3, folic acid levels, urine \& blood culture, thyroid \& complete lipid profile, viral screen including hepatitis B, C, HIV were all essentially normal. The electrocardiogram and chest x-ray were 
normal. The stool examination on wet mount confirmed presence of ova of hookworm. The live worms which were coming out of umbilicus on microscopic examination were confirmed as hookworm. The ultra-sonogram and computed tomography scan of abdomen was normal. The computed tomography Fistulogram from umbilicus was unable to show any tract between umbilicus and intestine or any other part which could have proven to be passage for worms from umbilicus. The patient was treated with mebendazole $100 \mathrm{mg}$ twice daily for five days, but worms kept on coming through umbilicus, though their number reduced from twenty to twenty-five per day to five to seven per day. Then patient was put on albendazole \& ivermectin combination which led to further decrease in number of worms coming from umbilicus to one to two per day, followed by decrease in size of worms and ultimately after a gap of one week, no worms were seen coming out of umbilicus. The fever subsided but pain abdomen was persistent (Figure 1,2).

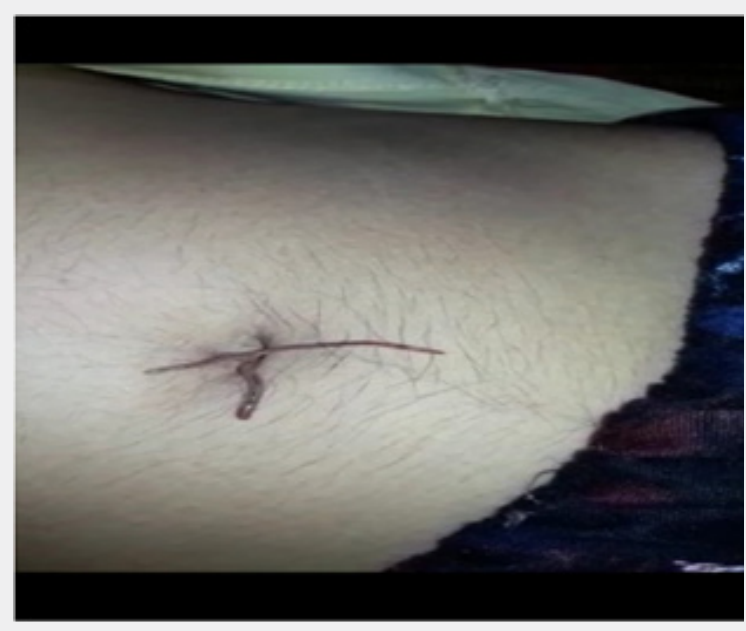

Figure 1: Multiple live hookworms coming out of umbilicus.

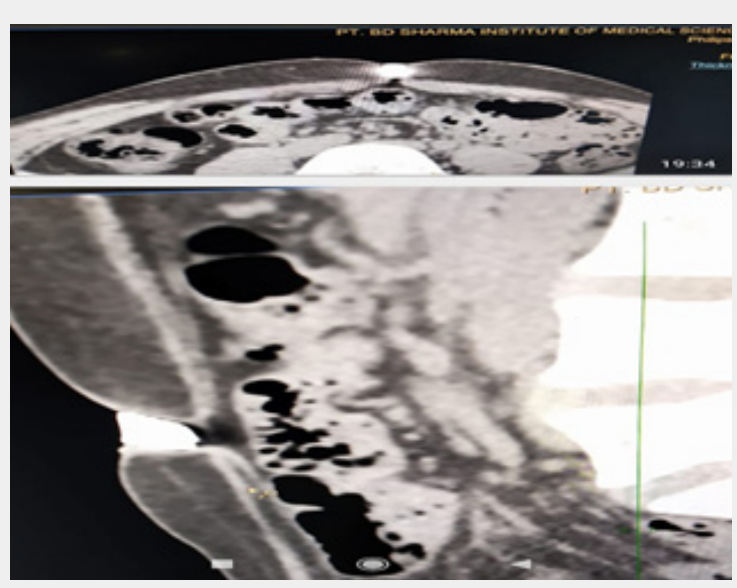

Figure 2: CT Fistulogram at umbilicus showing absence of any tract.

\section{Discussion}

Hookworm infection is endemic especially in tropical underdeveloped countries and one billion people are infected with it in the world $[3,4]$. It is mainly seen in rural areas, especially in children \& young women and is important cause of iron deficiency anemia. The adult worm resides in small intestine mucosa, thus leading to recurrent and persistent small hemorrhages and if ulcer formation is there, then it can cause upper gastrointestinal bleed, as it occurred in our case. The normal endoscopy may have been due to delay and use of proton pump inhibitors. These multiple small and large hemorrhages lead to iron deficiency anemia. The larvae usually enter through the skin, then migrates through the circulatory system, causing the pulmonary cycle, Löeffler's syndrome, pulmonary edema, alveolar lysis, fever, bronchitis and pneumonia. The filariform larvae can gain entry into body by ingestion of infected water or vegetables and by breastfeeding $[5,6]$. The disease severity depends on the parasite load, the nutritional and immunological status of the host. The presentation is with pain abdomen, generalized weakness, itching, 
anemia, hypoprotenemia, fever and in some cases with upper gastrointestinal bleed. Our case also presented with pain abdomen, fever, anemia, anorexia and upper gastrointestinal bleed which is consistent with documented literature. The history of passage of feculent material from umbilicus was suggestive of tract formed by worms between bowel and umbilicus but it being very small may have not been seen on Computed tomography fistulogram through umbilicus. The larvae migrate through circulatory system and can reach various organs of body. The adult worms have been reported in literature to come out through natural orifices like oral cavity, anal canal, nostrils and umbilicus but this is probably first case in world where multiple live worms have been reported to be exiting through umbilicus for many days.

The diagnosis takes account of patient's background, clinical manifestations, identification of parasite on stool examination and culture. Our case was confirmed on basis of identification of parasite on stool examination and examination of worms which were exiting through umbilicus. The drugs recommended for treatment includes mebendazole $100 \mathrm{mg}$ twice daily for 5 days, thiabendazole $10 \mathrm{mg} / \mathrm{kg}$ for 3 days, single dose of albendazole $400 \mathrm{mg}$, pyrantel pamoate and Ivermectin. We used mebendazole initially but due to partial response, albendazole \& Ivermectin combination was also given which lead to disappearance of worms coming out of umbilicus. The necessity of using dual therapy that too for prolonged duration in our case may have been due to high parasite load or presence of adult worms between intestine and umbilical opening where drugs effect is minimal. The proton pump inhibitors, antispasmodics, antibiotics and oral haematenics were used for symptomatic relief. The patient was managed conservatively and during stay of one week in indoor ward, there was no passage of worms from umbilicus, but mild pain abdomen persisted. The patient is on regular follow up for early detection of development of any features of peritonitis and management for the same.

\section{Conclusion}

The clinicians should be aware with atypical presentations of common diseases like hookworm infection, to minimize morbidity and mortality associated with such an easily preventable and treatable disease.

\section{References}

1. Gilles HM (1985) Selective primary health care: Strategies for control of disease in the developing world. XVII. Hookworm infection and anemia. Rev Infect Dis 7(1): 111-118.

2. Hotez PJ, Brooker S, Bethony JM, Bottazzi ME, Loukas A, et al. (2004) Hookworm infection. N Engl J Med 351(8): 799-807.

3. Roca C, Balanzó X, Sauca G, Fernández J, Boixeda R, et al. (2003) Uncinariasis importada por inmigrantes africanos: estudio de 285 casos. Med Clin Barc 121(4): 139-141.

4. Nair G, Cazorla E, Choque H, White A, Cabada M (2016) Infección masiva por Ancylostoma duodenale como causa de hemorragia intestinal y anemia severa. Soc Gastroenterol Perú 36(1): 90-92.

5. Valdivieso P, Cetraro D, Angulo D (2017) Hemorragia digestiva en neonato con uncinarias, hospital nacional san bartolomé. Rep Caso. Soc Gastroente-rol Perú 37(1): 82-86.

6. Kaminsky R (2000) Primer informe de Ancylostoma duodenale en honduras. Descripción clínica y parasitológica. Med Hond 68: 142-148. 